\title{
Dietary exposure and trends of exposure to nutrient elements iodine, iron, selenium and sodium from the 2003-4 New Zealand Total Diet Survey
}

\author{
Barbara M. Thomson ${ }^{1 *}$, Richard W. Vannoort ${ }^{1}$ and Roger M. Haslemore ${ }^{2}$ \\ ${ }^{1}$ Institute of Environmental Science and Research Ltd, PO Box 29 181, Christchurch, New Zealand \\ ${ }^{2} R$ J Hill Laboratories Ltd, Private Bag 3205, Hamilton, New Zealand \\ (Received 19 January 2007 - Revised 29 June 2007 - Accepted 4 July 2007)
}

The mean dietary exposure to the nutrient elements iodine, $\mathrm{Fe}$, Se and Na by eight age-sex groups of the New Zealand population was estimated from foods purchased and prepared as for consumption. A total of 968 samples comprising 121 foods were collected and analysed. Mean daily exposures were calculated from mean concentration levels of the selected nutrients in each food combined with simulated diets for a $25+-$ yearold male and female, a 19-24-year-old male, a 11-14-year-old boy and girl, a 5-6-year-old child, a 1-3-year-old toddler and a 6-12-month-old infant. Food concentrations and dietary exposures are reported and compared with nutrient reference values (for example, recommended daily intakes, adequate intakes or upper limits). Dietary iodine exposures for all age-sex groups were well below recommended levels and have steadily decreased since 1982, raising concern especially for the physical and mental development of infants and young children. Fe exposures meet the recommended daily intake for the average male and 11-14 year olds but are only about half that recommended for adult females. Se exposure is about $20 \%$ less than optimal for females. $\mathrm{Na}$ exposures, excluding discretionary salt, are above the acceptable exposure level for all age-sex groups, and exceed the upper intake limits for $25+$-year-old males, 19-24-year-old young males, and 11-14-year-old boys and girls by up to $125 \%$ for an average consumer.

Total diet surveys: Dietary exposure: Iodine: Iron: Selenium: Sodium

Iodine and Se are essential micronutrients that are deficient in New Zealand soils, resulting in low exposures for New Zealanders $^{1-3}$. Research has indicated an increasing prevalence of iodine deficiency in a sample of New Zealand children ${ }^{1}$. Iodine is involved in thyroid function, and affects both mental and physical development ${ }^{4,5}$. Se is an antioxidant that plays a part in the body's defence mechanisms ${ }^{6,7}$. Se deficiency, most probably in combination with other factors, is associated with heart and cartilage conditions known as Keshan and Kashin-Beck disease respectively. Se deficiency in combination with iodine deficiency has been reported to increase the risk of cretinism ${ }^{8}$.

$\mathrm{Fe}$ is an essential component of a number of proteins including $\mathrm{Hb}$, necessary for the transportation of $\mathrm{O}_{2}$ in the blood. Previous New Zealand Total Diet Surveys (NZTDS) in $1982^{9}, 1987-8^{10}$ and $1990-1^{11}$ reported low Fe exposures, ranging from 10 to $12 \mathrm{mg} / \mathrm{d}$, for women over 25 years relative to the current recommended dietary intake (RDI) of $18 \mathrm{mg} / \mathrm{d}$ for women of reproductive age ${ }^{8}$. New Zealand has allowed the fortification of several grain-based foods since the mid$1990 \mathrm{~s}$. No NZTDS has assessed Fe exposure since this fortification commenced.

$\mathrm{Na}$ is the principal cation in extracellular fluid with physiological roles closely linked to those of $\mathrm{K}$ and including the maintenance of extracellular fluid volume, acid-base balance, the active transport of molecules across cell membranes, transmission of nerve impulses and the contraction of muscles ${ }^{5,8}$. The kidneys maintain Na levels so that increased or decreased salt exposure does not usually increase the amount in the body ${ }^{5}$. The major adverse effect of increased sodium chloride exposure is elevated blood pressure, a risk factor for cardiovascular and renal diseases. Blood pressure increases progressively in a dose-dependent relationship with sodium chloride exposure, demonstrated in clinical trials published over 25 years from 1976 through to 2001 for $\mathrm{Na}$ exposures varying from $230 \mathrm{mg} / \mathrm{d}$ to $34500 \mathrm{mg} / \mathrm{d}^{8}$. Na is found in most foods as sodium chloride, commonly known as salt. Salt accounts for about $90 \%$ of total $\mathrm{Na}$ exposure for countries such as New Zealand and Australia ${ }^{12,13}$ with small amounts of $\mathrm{Na}$ exposure attributable to sodium bicarbonate, monosodium glutamate, sodium phosphate, sodium carbonate and sodium benzoate ${ }^{8,14}$. Mean $\mathrm{Na}$ exposures from previous NZTDS $^{10,11}$ for a 19-24-year-old young male, a female over 25 years and a 1-3-year-old toddler were at, or exceeded, the currently advocated upper intake level for $\mathrm{Na}^{8}$. Given the high prevalence of heart disease in New Zealand, Na exposure is a public health concern.

Total diet surveys are a recognised methodology used in a number of countries for assessing dietary exposures to agricultural compounds, heavy metals and nutrients ${ }^{15}$. Total diet surveys have been undertaken in New Zealand in $1974-5^{16,17}, 1982^{9}, 1987-8^{10}, 1990-1^{11,18,19}, 1997-8^{3,20}$ 
and $2003-4^{21}$. The four nutrient elements iodine, Fe, Se and $\mathrm{Na}$ were included in the suite of analytes measured in the 2003-4 NZTDS and food concentration data; exposure estimates for eight population groups and exposure trends are presented here.

\section{Experimental methods}

\section{Food selection, sampling and preparation}

The selection of foods for the 2003-4 NZTDS included 110 foods based on those of the previous NZTDS undertaken in 1997-8 with minor changes to reflect changing patterns of consumption. An additional eleven foods were added because they are significant sources of contaminants or agricultural compounds, such as oysters, mussels and liver, or are popular for specific sub-populations, especially children, such as flavoured milk and infant foods. The 121 foods of the 2003-4 NZTDS represent approximately $70 \%$ of the most commonly consumed foods in New Zealand. (a) national foods ( $n$ 63), which were not expected to exhibit any regional variability and included processed foods, such as biscuits, breakfast cereals and beverages, which are uniformly available throughout New Zealand. Up to four brands, selected on the basis of market share, were sampled in a single location in spring (October-November 2003) and autumn (May-June 2004);

(b) regional foods ( $n$ 58) that may vary in agricultural compound, contaminant and nutrient level depending on the location in which the food was produced. Regional foods included meat, milk, fruit and vegetables and were sampled in each of four locations in winter (JulyAugust 2003) and summer (January-February 2004).

Multiple units (two to twenty) or a minimum of $1 \mathrm{~kg}$ of each were collected on each sampling with the exception of cream $(500 \mathrm{ml})$, oats $(850 \mathrm{~g})$ and wine $(750 \mathrm{ml})$ where single units were considered sufficient. Foods were analysed on the basis of individual brands or region per season to give a total of four analyses for each food for each season. The following foods were purchased ready for consumption: battered fish, chicken takeaway, Chinese dish, corned beef, hot chips, meat pie and plain hamburger, and were mixed and chopped for analysis. All other foods were prepared to a 'table-ready' state before analysis. Package instructions were followed where appropriate (chocolate beverage, fish fingers, fruit drink, infant and follow-on formula, infant weaning foods, instant noodles, pizza and chicken soup). The following foods were boiled: beans, egg, kumara, pasta, peas, potatoes (peeled), pumpkin, rice, rolled oats, silverbeet and taro. Bacon, beef mince (ground beef), beef rump, lamb or mutton, lamb's liver, pork chops and sausages were fried without added fat. Fresh fish was grilled; potatoes (with skin) were cooked in a microwave. Vegetables were rinsed but fruits were not. Fruits that are normally consumed without peel, such as oranges and bananas, were peeled. Confectionery was melted in an equal weight of boiling water. All water used in food preparation was distilled and no salt was added to any foods at the time of cooking. Cooked or raw food samples were mixed, chopped, homogenised and frozen until analysis.

\section{Simulated diets and population groups}

As for previous NZTDS, food consumption data were derived from $14 \mathrm{~d}$ simulated typical diets for eight selected population age-sex groups: $25+$-year-old male; $25+$-year-old female; 19-24-year-old young male; 11-14-year-old boy; 11-14year-old girl; 5-6-year-old child; 1-3-year-old toddler; 6-12-month-old infant using the foods most commonly eaten by the New Zealand population plus a few specific foods for target populations such as infants and children ${ }^{22}$. These diets were based on national $24 \mathrm{~h}$ diet recall surveys conducted for New Zealanders aged 15 years and older ${ }^{23}$, children aged 5-14 years ${ }^{24}$ and on recent regional studies of young children $^{25}$ (C. Wall, personal communication, 2004) to represent an average consumer in each of the selected groups. A summary of the body weights, total weight of diet and estimated energy exposures of the simulated diets for each age-sex group in the 2003-4 NZTDS is given in Table 1, along with comparative data for 1997-8.

Table 1. Age-sex groups and body weights, total weight of diet and energy exposures of the simulated diets for the 2003-4 and 1997-98 New Zealand Total Diet Surveys

\begin{tabular}{|c|c|c|c|c|c|c|}
\hline \multirow[b]{2}{*}{ Age-sex group } & \multicolumn{2}{|c|}{ Body weight (kg) } & \multicolumn{2}{|c|}{ Total weight of diet $(\mathrm{g} / \mathrm{d})$} & \multicolumn{2}{|c|}{ Energy (MJ) } \\
\hline & $2003-4$ & $1997-98$ & $2003-4^{*}$ & $1997-98 \dagger$ & $2003-4^{*}$ & $1997-98 \dagger$ \\
\hline $25+$-year-old male & $82 \ddagger$ & 80 & 3461 & 2797 & 11.9 & $9 \cdot 8$ \\
\hline $25+$-year-old female & $70 \ddagger$ & 65 & 2779 & 2608 & $8 \cdot 2$ & $6 \cdot 8$ \\
\hline 19-24-year-old young male & $78 \ddagger$ & 70 & 3173 & 2817 & $13 \cdot 8$ & $11 \cdot 5$ \\
\hline 11-14-year-old boy & $54 \S$ & - & 2042 & - & $10 \cdot 4$ & - \\
\hline 11-14-year-old girl & $55 \S$ & - & 1790 & - & 8.5 & - \\
\hline 5-6-year-old child & $23 \S$ & $20 \|$ & 1633 & $1961 \|$ & $7 \cdot 2$ & $6 \cdot 6 \|$ \\
\hline 1-3-year-old toddler & 139 & - & 1275 & - & $5 \cdot 2$ & 4.7 \\
\hline $6-12$-month-old infant & 99 & - & 1044 & - & $3 \cdot 8$ & - \\
\hline
\end{tabular}

*Derived from 1997 National Nutrition Survey data 22 .

†Derived from 1990 Life in New Zealand survey data ${ }^{47}$.

$\ddagger$ National Nutrition Survey, $1997^{23}$.

$\S$ Children's Nutrition Survey, $2002^{24}$

|| 4-6 year olds.

ๆ National Health and Medical Research Council/Ministry of Health, $2006^{8}$. 


\section{Chemical analysis of analytes}

Accurately weighed sub-samples $(0.5-2.5 \mathrm{~g})$ of the homogenised foods were prepared for analysis as follows. Samples for the determination of $\mathrm{Na}$ and $\mathrm{Fe}$ were digested with nitric and hydrochloric acids and the acid digests were then analysed by inductively coupled plasma-optical emission spectroscopy. Samples for iodine and Se were digested with tetramethylammonium hydroxide ${ }^{26}$ and the alkaline digests were then analysed by inductively coupled plasma MS. The limit of detection for iodine, $\mathrm{Fe}, \mathrm{Se}$ and $\mathrm{Na}$ varied from 0.001 to $50 \mathrm{mg} / \mathrm{kg}$ depending on the element, the fat and the water content of the food.

\section{Quality assurance}

A comprehensive set of quality-assurance samples, including spikes, duplicates, certified reference materials and blanks were included with each batch of samples to ensure confidence in the methodology and robustness of the results. The analytical variability for each nutrient was expressed as the CV (standard deviation of the results divided by mean $\times 100 \%$ ) based on 84-104 duplicates covering the range of food matrices. Spiking concentrations were varied depending on the nutrient: iodine, $0.006 \mathrm{mg} / \mathrm{kg}$; Fe, $0.3 \mathrm{mg} / \mathrm{kg}$; Se, $0.006 \mathrm{mg} / \mathrm{kg} ; \mathrm{Na}, 30 \mathrm{mg} / \mathrm{kg}$.

\section{Exposure estimations}

The estimates of dietary exposures were calculated by using the arithmetic mean concentration of the nutrient element in food multiplied by the daily weight for each food consumed (derived from 2-week simulated diets) for each age-sex group. A 'not detected' result was assigned a level of half the limit of detection for the calculation of mean concentration.

\section{Results and discussion}

\section{Analytical quality assurance}

The mean recovery of nutrients from spiked food matrices ranged from $95 \%$ for $\mathrm{Fe}$ to $102 \%$ for iodine (Table 2). Analytical variability was a challenge given the number of matrices and range of concentration levels. The variability, expressed as $\mathrm{CV}$ and calculated across all duplicate samples for each nutrient, was 21, 41, 17 and 9 for iodine, $\mathrm{Fe}, \mathrm{Se}$ and $\mathrm{Na}$ respectively. The high variability observed for Fe was due to the seven samples with $\mathrm{Fe}$ concentrations greater than $30 \mathrm{mg} / \mathrm{kg}$. For the ninety-seven duplicates where the Fe concentration was less than $30 \mathrm{mg} / \mathrm{kg}$ the $\mathrm{CV}$ was 11 . The accuracy of the determinations, based on recovery from certified or reference materials, was better than $85 \%$ except for Se in bovine muscle and $\mathrm{Fe}$ in rice flour, apple leaves and the oyster reference material that were as low as $69 \%$ (Table 2). $\mathrm{Fe}$ was known to be a difficult element to obtain good recoveries from some certified reference materials unless hydrofluoric acid was used to digest these materials.

\section{Nutrient concentrations of foods}

The mean, minimum, maximum and proportion of samples containing nutrients at concentrations above the limit of detection are shown in Table 3.
Iodine. The iodine concentrations of most foods $(65 \%)$ were less than $0.020 \mathrm{mg} / \mathrm{kg}$. However, three foods had elevated iodine content, namely one of four brands of soya milk $(9.14 \mathrm{mg} / \mathrm{kg})$, mussels $(3.34 \mathrm{mg} / \mathrm{kg})$ and oysters $(1.38 \mathrm{mg} / \mathrm{kg})$. The latter two were in the expected range, but soya milk normally has less than $0.01 \mathrm{mg}$ iodine $/ \mathrm{kg}$. In this instance, the 2003-4 NZTDS helped identify an unexpectedly and unacceptably high iodine concentration in the New Zealand food supply. The manufacturer was informed and the product reformulated.

Iron. Most foods contained less than $50 \mathrm{mg} \mathrm{Fe} / \mathrm{kg}$. The highest concentration of $\mathrm{Fe}$ was found in yeast extract and lamb's liver at 446 and $435 \mathrm{mg} / \mathrm{kg}$, respectively. A number of cereal products, namely bran flake cereal, cornflakes, cerealbased infant weaning food and wheat biscuit cereals, had maximum Fe levels above $100 \mathrm{mg} / \mathrm{kg}$. Mussels and oysters also had maximum $\mathrm{Fe}$ concentrations above $100 \mathrm{mg} / \mathrm{kg}$.

Selenium. The concentration of Se in the 121 foods of the 2003-4 NZTDS ranged from $<0.002$ to $0.64 \mathrm{mg} / \mathrm{kg}$, with the highest level measured in mussels. Seafoods in general had higher concentrations of Se than other foods. Animal products (eggs, chicken, pork, beef and lamb) appeared to have Se levels that were intermediate between previous NZTDS in 1997-8 and 1987-8 studies. The Se concentrations for 2003-4 NZTDS foods were all lower than concentrations reported for either Australia, USA or both ${ }^{27,28}$ but not dissimilar to levels reported in the 1st French Total Diet Study ${ }^{29}$.

Sodium. The concentration of $\mathrm{Na}$ in the 121 foods of the 2003-4 NZTDS ranged from $<10$ to $42000 \mathrm{mg} / \mathrm{kg}$, with the highest level measured in a yeast extract. Mean Na concentrations varied markedly between and within food groups. The lowest $\mathrm{Na}$ concentrations were generally in the fruit, vegetable and infant weaning food groups. It is also apparent that much higher $\mathrm{Na}$ concentrations are found in processed compared with unprocessed foods. For example, tomatoes contained $36 \mathrm{mg} \mathrm{Na} / \mathrm{kg}$, whereas tomato sauce contained $7070 \mathrm{mg} / \mathrm{kg}$. Similarly, pork chops contained $840 \mathrm{mg} \mathrm{Na} / \mathrm{kg}$, while bacon contained $15300 \mathrm{mg} / \mathrm{kg}$; and cream $(250 \mathrm{mg} /$ $\mathrm{kg}$ ) can be compared with cheese $(6300 \mathrm{mg} \mathrm{Na} / \mathrm{kg})$.

\section{Exposure calculations}

A summary of mean daily exposures of iodine, $\mathrm{Fe}, \mathrm{Se}$ and $\mathrm{Na}$ for each age-sex group, based on mean concentration and average consumption is shown in Table 4 with nutrient reference values ${ }^{8}$. The simulated diets used for estimating nutrient exposures in the present study were based on average energy consumption figures and therefore exposure estimates are limited to the population average. But generally, high consumers at 95th percentile levels may approximate three times the population average consumption figure for individual foods, and up to twice the total amount consumed by the population as a whole ${ }^{30}$. Nutrient exposure for these consumers will therefore be up to twice that of the average population.

Iodine. Daily exposures of iodine were calculated for both normal and high levels of iodine in soya milk. There was little difference between lower and upper bound iodine exposure estimates and mean estimates of iodine in each age-sex group. Assuming normal levels of iodine in soya milk, the estimated mean exposures in the 2003-4 NZTDS were significantly lower than the RDI for all age-sex groups. Iodine 
Table 2. Analytical quality-assurance results for spikes, duplicates and certified reference materials (CRM) (Mean values and two standard deviations)

\begin{tabular}{|c|c|c|c|c|c|c|c|c|c|c|c|c|c|c|c|c|}
\hline & \multicolumn{4}{|c|}{ lodine } & \multicolumn{4}{|c|}{$\mathrm{Fe}$} & \multicolumn{4}{|c|}{$\mathrm{Se}$} & \multicolumn{4}{|c|}{$\mathrm{Na}$} \\
\hline & \multicolumn{2}{|c|}{ Cert (mg/kg) } & \multicolumn{2}{|c|}{ Meas $(\mathrm{mg} / \mathrm{kg})$} & \multicolumn{2}{|c|}{ Cert (mg/kg) } & \multicolumn{2}{|c|}{ Meas $(\mathrm{mg} / \mathrm{kg})$} & \multicolumn{2}{|c|}{ Cert $(\mathrm{mg} / \mathrm{kg})$} & \multicolumn{2}{|c|}{ Meas $(\mathrm{mg} / \mathrm{kg})$} & \multicolumn{2}{|c|}{ Cert (mg/kg) } & \multicolumn{2}{|c|}{ Meas (mg/kg) } \\
\hline & Mean & $2 \mathrm{SD}$ & Mean & $2 \mathrm{SD}$ & Mean & $2 \mathrm{SD}$ & Mean & $2 \mathrm{SD}$ & Mean & $2 \mathrm{SD}$ & Mean & $2 \mathrm{SD}$ & Mean & $2 \mathrm{SD}$ & Mean & $2 \mathrm{SD}$ \\
\hline \multicolumn{17}{|l|}{ Percentage spike recovery ${ }^{*}$} \\
\hline Mean & & & 102 & & & & 95 & & & & 100 & & & & 96 & \\
\hline Range & & & $86-116$ & & & & $34-142$ & & & & $87-114$ & & & & $69-112$ & \\
\hline$n$ & & & 78 & & & & 95 & & & & 79 & & & & 83 & \\
\hline Percentage $\mathrm{CV} \dagger$ & & & 21 & & & & 41 & & & & 17 & & & & 9 & \\
\hline $\begin{array}{l}\text { Percentage CV } n \\
\text { CRM } \neq\end{array}$ & & & 84 & & & & 104 & & & & 84 & & & & 99 & \\
\hline NIST1549QC & 3.38 & 0.02 & 3.34 & 0.30 & 1.78 & 0.10 & 1.3 & 1.6 & 0.11 & 0.01 & 0.10 & 0.02 & 4970 & 100 & 4930 & 348 \\
\hline SRM 1577b Bovine liver & - & - & - & - & 184 & 15 & 177 & 10 & 0.73 & 0.06 & 0.69 & 0.08 & 2420 & 60 & 2379 & 238 \\
\hline RM 8414 Bovine muscle & 0.035 & 0.012 & 0.033 & 0.016 & 71.2 & 9.2 & $65 \cdot 2$ & $5 \cdot 2$ & 0.08 & 0.01 & 0.061 & 0.018 & 2100 & 80 & 2122 & 208 \\
\hline SRM 1568a Rice flour & - & - & - & - & 7.4 & 0.9 & $5 \cdot 8$ & 4.6 & 0.38 & 0.04 & 0.33 & 0.04 & - & - & - & - \\
\hline SRM 1515 Apple leaves & - & - & 0.6 & - & 83 & 5 & 56 & 16 & 0.05 & 0.009 & - & - & - & - & - & - \\
\hline SRM 1566b Oyster & - & - & - & - & 206 & 7 & 168 & 24 & 2.06 & 0.15 & 1.75 & 0.14 & 3297 & 53 & 3101 & 298 \\
\hline
\end{tabular}

\section{Cert, certified value; Meas, measured value.}

Spiking concentrations for various food matrices were: I, $0.006 \mathrm{mg} / \mathrm{kg} ; \mathrm{Fe}, 0.3 \mathrm{mg} / \mathrm{kg} ; \mathrm{Se}, 0.006 \mathrm{mg} / \mathrm{kg} ; \mathrm{Na}, 30 \mathrm{mg} / \mathrm{kg}$

$\dagger$ Mean precision based on duplicates spread across a range of food matrices.

F Measured results for CRM have not been corrected for moisture content. The uncertainty for certified and measured values is stated at a $95 \% \mathrm{Cl}$ (i.e. \pm 2 sD). 
NS British Journal of Nutrition

Table 3. Mean concentrations of iodine, iron, selenium and sodium in 'table-ready' foods*

(Mean, minimum (Min) and maximum(Max) values)

\begin{tabular}{|c|c|c|c|c|c|c|c|c|c|c|c|c|c|c|c|c|}
\hline \multirow[b]{2}{*}{ Foods $(n 8)$} & \multicolumn{4}{|c|}{ lodine $(\mathrm{mg} / \mathrm{kg})$} & \multicolumn{4}{|c|}{$\mathrm{Fe}(\mathrm{mg} / \mathrm{kg})$} & \multicolumn{4}{|c|}{ Se $(\mathrm{mg} / \mathrm{kg})$} & \multicolumn{4}{|c|}{$\mathrm{Na}(\mathrm{mg} / \mathrm{kg})$} \\
\hline & $\begin{array}{l}\text { No. }> \\
\text { LOD }\end{array}$ & Mean & Min & $\operatorname{Max}$ & $\begin{array}{l}\text { No. }> \\
\text { LOD }\end{array}$ & Mean & Min & $\operatorname{Max}$ & $\begin{array}{l}\text { No. }> \\
\text { LOD }\end{array}$ & Mean & Min & $\operatorname{Max}$ & $\begin{array}{l}\text { No. }> \\
\text { LOD }\end{array}$ & Mean & Min & $\operatorname{Max}$ \\
\hline Apple & 2 & 0.0020 & $<0.002$ & 0.007 & 8 & 0.9 & 0.7 & 1.0 & 0 & 0.0020 & $<0.004$ & $<0.004$ & 6 & 12 & $<10$ & 27 \\
\hline Apple-based juice & 8 & 0.0089 & 0.002 & 0.047 & 6 & 0.5 & $<1.0$ & $2 \cdot 8$ & 0 & 0.0010 & $<0.002$ & $<0.002$ & 7 & 31 & $<5$ & 77 \\
\hline Apricot, canned & 8 & 0.0149 & 0.009 & 0.024 & 8 & $2 \cdot 3$ & 1.4 & 3.4 & 0 & 0.0020 & $<0.004$ & $<0.004$ & 6 & 22 & $<10$ & 84 \\
\hline Avocado & 0 & 0.0010 & $<0.002$ & $<0.002$ & 8 & $5 \cdot 1$ & 3.6 & $7 \cdot 2$ & 4 & 0.0053 & $<0.004$ & 0.012 & 8 & 86 & 52 & 157 \\
\hline Bacon, fried & 8 & 0.0106 & 0.008 & 0.014 & 8 & $6 \cdot 1$ & 5.4 & $7 \cdot 0$ & 8 & 0.1381 & 0.118 & 0.180 & 8 & 15250 & 12600 & 18900 \\
\hline Banana, peeled & 2 & 0.0014 & $<0.002$ & 0.003 & 8 & $2 \cdot 2$ & 1.7 & $2 \cdot 8$ & 0 & 0.0020 & $<0.004$ & $<0.004$ & 0 & 5 & $<10$ & $<10$ \\
\hline Beans, boiled & 1 & 0.0014 & $<0.002$ & 0.004 & 8 & $5 \cdot 3$ & 3.9 & $7 \cdot 2$ & 0 & 0.0020 & $<0.004$ & $<0.004$ & 0 & 5 & $<10$ & $<10$ \\
\hline $\begin{array}{l}\text { Beans, baked, } \\
\text { canned }\end{array}$ & 8 & 0.0158 & 0.011 & 0.026 & 8 & 11.5 & $9 \cdot 2$ & $14 \cdot 6$ & 8 & 0.0238 & 0.007 & 0.037 & 8 & 4284 & 3190 & 5150 \\
\hline Beef, mince, fried & 8 & 0.0101 & 0.005 & 0.031 & 8 & $24 \cdot 0$ & $17 \cdot 7$ & $29 \cdot 3$ & 8 & 0.0570 & 0.028 & 0.077 & 8 & 811 & 559 & 1240 \\
\hline Beef, rump, fried & 8 & 0.0071 & 0.004 & 0.010 & 8 & $33 \cdot 7$ & 24.9 & $47 \cdot 0$ & 8 & 0.0764 & 0.042 & 0.140 & 8 & 671 & 561 & 776 \\
\hline Beer & 6 & 0.0127 & $<0.001$ & 0.046 & 0 & 0.1 & $<0.1$ & $<0.1$ & 0 & 0.0010 & $<0.002$ & $<0.002$ & 8 & 18 & 13 & 29 \\
\hline Beetroot, canned & 8 & 0.0225 & 0.016 & 0.030 & 8 & 3.3 & $2 \cdot 0$ & 4.8 & 0 & 0.0020 & $<0.004$ & $<0.004$ & 8 & 1567 & 349 & 3430 \\
\hline Biscuit, chocolate & 8 & 0.0551 & 0.018 & 0.173 & 8 & $30 \cdot 2$ & $10 \cdot 0$ & $53 \cdot 3$ & 3 & 0.0178 & $<0.02$ & 0.043 & 8 & 1624 & 610 & 2795 \\
\hline Biscuit, cracker & 1 & 0.0081 & $<0.01$ & 0.030 & 8 & $18 \cdot 3$ & $10 \cdot 0$ & 38.0 & 7 & 0.0963 & $<0.02$ & 0.370 & 8 & 4640 & 2410 & 7680 \\
\hline $\begin{array}{l}\text { Biscuits, plain } \\
\text { sweet }\end{array}$ & 1 & 0.0069 & $<0.01$ & 0.020 & 8 & $10 \cdot 8$ & $6 \cdot 0$ & $19 \cdot 0$ & 4 & 0.0413 & $<0.02$ & 0.160 & 8 & 3620 & 2730 & 6870 \\
\hline $\begin{array}{l}\text { Bran flake cereal, } \\
\text { mixed }\end{array}$ & 2 & 0.0100 & $<0.01$ & 0.040 & 8 & 71.4 & $36 \cdot 0$ & $140 \cdot 0$ & 8 & 0.0875 & 0.050 & 0.130 & 7 & 3147 & $<50$ & 7150 \\
\hline Bread, mixed grain & 7 & 0.0116 & $<0.005$ & 0.025 & 8 & $15 \cdot 9$ & $12 \cdot 7$ & $26 \cdot 2$ & 7 & 0.0381 & $<0.010$ & 0.093 & 8 & 4469 & 3900 & 5210 \\
\hline Bread, wheatmeal & 3 & 0.0048 & $<0.005$ & 0.011 & 8 & 17.5 & 14.6 & 19.5 & 4 & 0.0438 & $<0.010$ & 0.131 & 8 & 5006 & 4520 & 5910 \\
\hline Bread, white & 2 & 0.0032 & $<0.005$ & 0.007 & 8 & $12 \cdot 9$ & 7.6 & $39 \cdot 2$ & 4 & 0.0354 & $<0.010$ & 0.102 & 8 & 5063 & 4710 & 5470 \\
\hline $\begin{array}{l}\text { Broccoli or cauli- } \\
\text { flower }\end{array}$ & 1 & 0.0013 & $<0.002$ & 0.003 & 8 & 4.5 & 3.0 & 5.9 & 4 & 0.0060 & $<0.004$ & 0.016 & 8 & 54 & 39 & 77 \\
\hline Butter & 5 & 0.0096 & $<0.010$ & 0.016 & 0 & 0.5 & $<1.0$ & $<1.0$ & 0 & 0.0100 & $<0.020$ & $<0.020$ & 8 & 5586 & 5370 & 5960 \\
\hline Cabbage & 0 & 0.0010 & $<0.002$ & $<0.002$ & 8 & $2 \cdot 7$ & 1.7 & $4 \cdot 3$ & 2 & 0.0035 & $<0.004$ & 0.009 & 8 & 82 & 35 & 145 \\
\hline $\begin{array}{l}\text { Caffeinated bever- } \\
\text { age }\end{array}$ & 4 & 0.0013 & $<0.001$ & 0.003 & 0 & 0.1 & $<0.1$ & $<0.1$ & 0 & 0.0010 & $<0.002$ & $<0.002$ & 8 & 605 & 176 & 956 \\
\hline Cake & 8 & 0.1300 & 0.097 & 0.206 & 8 & $9 \cdot 3$ & $6 \cdot 8$ & $11 \cdot 2$ & 8 & 0.0738 & 0.060 & 0.089 & 8 & 3680 & 3270 & 3870 \\
\hline Capsicum & 0 & 0.0010 & $<0.002$ & $<0.002$ & 8 & $2 \cdot 1$ & 1.4 & 2.9 & 0 & 0.0020 & $<0.004$ & $<0.004$ & 4 & 9 & $<10$ & 13 \\
\hline Carbonated drink & 6 & 0.0020 & $<0.001$ & 0.005 & 0 & $0 \cdot 1$ & $<0.1$ & $<0.1$ & 0 & 0.0010 & $<0.002$ & $<0.002$ & 8 & 44 & 29 & 56 \\
\hline Carrot & 5 & 0.0044 & $<0.002$ & 0.018 & 8 & 1.4 & $1 \cdot 1$ & $2 \cdot 1$ & 1 & 0.0025 & $<0.004$ & 0.006 & 8 & 364 & 213 & 554 \\
\hline Celery & 6 & 0.0095 & $<0.002$ & 0.044 & 8 & 1.5 & 0.7 & $5 \cdot 0$ & 0 & 0.0020 & $<0.004$ & $<0.004$ & 8 & 455 & 175 & 843 \\
\hline Cheese & 8 & 0.0625 & 0.050 & 0.070 & 5 & $1 \cdot 1$ & $<1.0$ & $2 \cdot 0$ & 8 & 0.0550 & 0.050 & 0.070 & 8 & 6304 & 5840 & 6940 \\
\hline Chicken, fried & 8 & 0.0150 & 0.003 & 0.043 & 8 & 8.3 & $4 \cdot 3$ & 14.5 & 8 & 0.1990 & 0.101 & 0.317 & 8 & 3521 & 850 & 7320 \\
\hline Chicken takeaway & 8 & 0.0316 & 0.004 & 0.116 & 8 & $6 \cdot 8$ & $5 \cdot 7$ & $10 \cdot 1$ & 8 & 0.1091 & 0.055 & 0.135 & 8 & 7309 & 6380 & 9790 \\
\hline Chinese dish & 8 & 0.0453 & 0.008 & 0.240 & 8 & $4 \cdot 7$ & $3 \cdot 8$ & $5 \cdot 8$ & 8 & 0.0423 & 0.026 & 0.061 & 8 & 4316 & 3590 & 5430 \\
\hline $\begin{array}{l}\text { Chocolate bever- } \\
\text { age }\end{array}$ & 6 & 0.0133 & $<0.001$ & 0.031 & 8 & $3 \cdot 7$ & 0.5 & 7.4 & 2 & 0.0015 & $<0.002$ & 0.003 & 6 & 57 & $<5$ & 106 \\
\hline $\begin{array}{l}\text { Chocolate, plain } \\
\text { milk }\end{array}$ & 8 & 0.1525 & 0.070 & 0.510 & 8 & $15 \cdot 5$ & $10 \cdot 0$ & $24 \cdot 0$ & 1 & 0.0113 & $<0.020$ & 0.020 & 8 & 706 & 556 & 825 \\
\hline $\begin{array}{l}\text { Coffee beans, } \\
\text { ground }\end{array}$ & 0 & 0.0008 & $<0.001$ & $<0.002$ & 2 & $0 \cdot 1$ & $<0.1$ & 0.3 & 0 & 0.0015 & $<0.002$ & $<0.004$ & 0 & 4 & $<5$ & $<10$ \\
\hline Coffee, instant & 2 & 0.0006 & $<0.001$ & 0.001 & 8 & 0.6 & 0.3 & 1.0 & 0 & 0.0010 & $<0.002$ & $<0.002$ & 3 & 4 & $<5$ & 7 \\
\hline Confectionery & 2 & 0.0075 & $<0.010$ & 0.020 & 1 & 0.6 & $<1.0$ & 1.0 & 0 & 0.0100 & $<0.020$ & $<0.020$ & 5 & 205 & $<50$ & 580 \\
\hline Corn, canned & 8 & 0.0081 & 0.005 & 0.012 & 8 & $2 \cdot 8$ & 1.9 & 3.4 & 4 & 0.0069 & $<0.004$ & 0.024 & 8 & 1330 & 953 & 2470 \\
\hline
\end{tabular}


NS British Journal of Nutrition

Table 3. Continued

\begin{tabular}{|c|c|c|c|c|c|c|c|c|c|c|c|c|c|c|c|c|}
\hline \multirow[b]{2}{*}{ Foods ( $(n)$} & \multicolumn{4}{|c|}{ lodine (mg/kg) } & \multicolumn{4}{|c|}{$\mathrm{Fe}(\mathrm{mg} / \mathrm{kg})$} & \multicolumn{4}{|c|}{ Se $(\mathrm{mg} / \mathrm{kg})$} & \multicolumn{4}{|c|}{$\mathrm{Na}(\mathrm{mg} / \mathrm{kg})$} \\
\hline & $\begin{array}{l}\text { No. }> \\
\text { LOD }\end{array}$ & Mean & Min & $\operatorname{Max}$ & $\begin{array}{r}\text { No. }> \\
\text { LOD }\end{array}$ & Mean & Min & $\operatorname{Max}$ & $\begin{array}{l}\text { No. }> \\
\text { LOD }\end{array}$ & Mean & Min & $\operatorname{Max}$ & $\begin{array}{l}\text { No. }>> \\
\text { LOD }\end{array}$ & Mean & Min & $\operatorname{Max}$ \\
\hline $\begin{array}{l}\text { Corned beef, pre- } \\
\text { cooked }\end{array}$ & 8 & 0.0250 & 0.015 & 0.043 & 8 & $19 \cdot 2$ & $15 \cdot 6$ & $21 \cdot 0$ & 8 & 0.0446 & 0.033 & 0.052 & 8 & 8704 & 7290 & 11300 \\
\hline Cornflakes & 1 & 0.0069 & $<0.010$ & 0.020 & 8 & $81 \cdot 4$ & $6 \cdot 0$ & 141.0 & 2 & 0.0188 & $<0.020$ & 0.060 & 8 & 7104 & 4460 & 9600 \\
\hline Courgette & 2 & 0.0015 & $<0.002$ & 0.004 & 8 & 3.1 & $2 \cdot 0$ & 5.6 & 2 & 0.0031 & $<0.004$ & 0.008 & 0 & 5 & $<10$ & $<10$ \\
\hline Cream & 8 & 0.0609 & 0.028 & 0.182 & 8 & 0.4 & 0.2 & 0.5 & 8 & 0.0055 & 0.004 & 0.007 & 8 & 249 & 224 & 268 \\
\hline Cucumber & 1 & 0.0014 & $<0.002$ & 0.004 & 8 & 1.0 & 0.6 & 1.4 & 0 & 0.0020 & $<0.004$ & $<0.004$ & 6 & 20 & $<10$ & 32 \\
\hline Dairy dessert & 8 & 0.0665 & 0.045 & 0.093 & 6 & 1.3 & $<0.2$ & $3 \cdot 2$ & 6 & 0.0060 & $<0.004$ & 0.009 & 8 & 569 & 493 & 723 \\
\hline Egg, boiled & 8 & 0.5192 & 0.432 & 0.690 & 8 & $9 \cdot 3$ & 6.5 & $12 \cdot 2$ & 8 & 0.2685 & 0.242 & 0.309 & 8 & 1431 & 1340 & 1530 \\
\hline Fish fingers, baked & 8 & 0.0319 & 0.020 & 0.045 & 8 & 3.9 & 2.5 & 5.4 & 8 & 0.2545 & 0.189 & 0.397 & 8 & 3783 & 2880 & 5670 \\
\hline Fish, battered & 8 & 0.1658 & 0.025 & 0.456 & 8 & $2 \cdot 8$ & $2 \cdot 1$ & 3.9 & 8 & $0 \cdot 3014$ & 0.188 & 0.420 & 8 & 2009 & 1520 & 2470 \\
\hline Fish, canned & 8 & 0.1299 & 0.078 & 0.213 & 8 & $9 \cdot 6$ & $7 \cdot 1$ & 14.7 & 8 & 0.5055 & 0.311 & 0.772 & 8 & 2902 & 477 & 6130 \\
\hline Fish, fresh, grilled & 8 & 0.2159 & 0.117 & 0.372 & 8 & $2 \cdot 3$ & 1.4 & 3.3 & 8 & 0.4920 & 0.370 & 0.585 & 8 & 738 & 542 & 931 \\
\hline Fruit drink & 1 & 0.0007 & $<0.001$ & 0.002 & 0 & 0.1 & $<0.1$ & $<0.1$ & 0 & 0.0010 & $<0.002$ & $<0.002$ & 8 & 172 & 70 & 335 \\
\hline Grapes & 6 & 0.0049 & $<0.002$ & 0.019 & 8 & 3.4 & 2.4 & 4.9 & 0 & 0.0020 & $<0.004$ & $<0.004$ & 6 & 26 & $<10$ & 69 \\
\hline Ham & 8 & 0.0371 & 0.023 & 0.056 & 8 & $7 \cdot 7$ & $6 \cdot 8$ & $9 \cdot 0$ & 8 & $0 \cdot 1416$ & 0.109 & 0.170 & 8 & 13275 & 12100 & 15000 \\
\hline $\begin{array}{l}\text { Hamburger, plain, } \\
\text { pre-cooked }\end{array}$ & 8 & 0.0233 & 0.005 & 0.082 & 8 & 14.9 & $11 \cdot 2$ & $17 \cdot 2$ & 8 & 0.0935 & 0.037 & 0.197 & 8 & 4745 & 3760 & 6870 \\
\hline Honey & 1 & 0.0056 & $<0.010$ & 0.010 & 5 & 1.2 & $<1.0$ & $2 \cdot 0$ & 0 & 0.0100 & $<0.020$ & $<0.020$ & 1 & 29 & $<50$ & 60 \\
\hline Ice-cream & 8 & 0.0680 & 0.043 & 0.117 & 5 & 0.3 & $<0.2$ & 0.5 & 5 & 0.0069 & $<0.004$ & 0.015 & 8 & 459 & 373 & 647 \\
\hline $\begin{array}{l}\text { Infant and follow- } \\
\text { on formula }\end{array}$ & 8 & 0.0786 & 0.030 & 0.109 & 8 & 8.5 & $4 \cdot 6$ & $12 \cdot 9$ & 6 & 0.0108 & $<0.002$ & 0.027 & 8 & 219 & 98 & 337 \\
\hline IWF, cereal based & 8 & 0.0249 & 0.004 & 0.061 & 7 & $47 \cdot 0$ & $<0.2$ & 129.0 & 5 & 0.0075 & $<0.004$ & 0.020 & 7 & 130 & $<10$ & 285 \\
\hline $\begin{array}{l}\text { IWF, custard and } \\
\text { fruit dish }\end{array}$ & 8 & 0.0614 & 0.006 & 0.112 & 8 & 3.7 & 0.9 & 9.6 & 6 & 0.0101 & $<0.004$ & 0.024 & 8 & 154 & $12 \cdot 5$ & 350 \\
\hline IWF, savoury & 6 & 0.0195 & $<0.002$ & 0.062 & 8 & $5 \cdot 4$ & 3.8 & 7.5 & 7 & 0.0098 & $<0.004$ & 0.019 & 8 & 270 & 113 & 382 \\
\hline Jam & 0 & 0.0050 & $<0.010$ & $<0.010$ & 6 & 1.0 & $<1.0$ & $2 \cdot 0$ & 0 & 0.0100 & $<0.020$ & $<0.020$ & 5 & 160 & $<50$ & 381 \\
\hline Kiwi fruit & 0 & 0.0010 & $<0.002$ & $<0.002$ & 8 & $2 \cdot 2$ & 1.7 & $2 \cdot 7$ & 3 & 0.0048 & $<0.004$ & 0.013 & 5 & 12 & $<10$ & 22 \\
\hline Kumara, boiled & 4 & 0.0029 & $<0.002$ & 0.010 & 8 & 3.9 & $3 \cdot 3$ & 4.8 & 0 & 0.0020 & $<0.004$ & $<0.004$ & 8 & 152 & 78 & 218 \\
\hline Lamb or multon, fried & 8 & 0.0323 & 0.006 & 0.175 & 8 & $26 \cdot 3$ & $16 \cdot 3$ & $35 \cdot 2$ & 8 & 0.0533 & 0.038 & 0.081 & 8 & 934 & 840 & 1090 \\
\hline Lamb's liver, fried & 8 & 0.0684 & 0.048 & 0.151 & 8 & $147 \cdot 1$ & 62.5 & 435.0 & 8 & 0.1975 & 0.094 & 0.263 & 8 & 942 & 822 & 1070 \\
\hline Lettuce & 4 & 0.0068 & $<0.002$ & 0.040 & 8 & 2.7 & $1 \cdot 1$ & 4.1 & 1 & 0.0029 & $<0.004$ & 0.010 & 8 & 30 & 21 & 55 \\
\hline Margarine & 0 & 0.0050 & $<0.010$ & $<0.010$ & 4 & 2.0 & $<1.0$ & 4.0 & NR & 0.0059 & 0.006 & 0.006 & 8 & 5409 & 3450 & 6550 \\
\hline Meat pie & 8 & 0.0076 & 0.004 & 0.011 & 8 & 8.8 & $7 \cdot 6$ & $11 \cdot 2$ & 8 & 0.0286 & 0.010 & 0.056 & 8 & 4599 & 3840 & 5320 \\
\hline Melons & 0 & 0.0010 & $<0.002$ & $<0.002$ & 8 & 1.7 & $1 \cdot 3$ & $2 \cdot 2$ & 0 & 0.0020 & $<0.004$ & $<0.004$ & 8 & 77 & 38 & 137 \\
\hline Milk, $0.5 \%$ fat & 8 & 0.0963 & 0.036 & 0.312 & 8 & 0.2 & $0 \cdot 1$ & 0.2 & 8 & 0.0081 & 0.005 & 0.013 & 8 & 391 & 357 & 430 \\
\hline Milk, $3.25 \%$ fat & 8 & 0.0858 & 0.041 & 0.235 & 8 & 0.2 & 0.2 & 0.2 & 8 & 0.0066 & 0.003 & 0.010 & 8 & 383 & 347 & 414 \\
\hline Milk, flavoured & 8 & 0.0596 & 0.042 & 0.101 & 8 & 0.9 & 0.5 & $2 \cdot 1$ & 7 & 0.0058 & $<0.002$ & 0.009 & 8 & 410 & 334 & 611 \\
\hline Muesli & 3 & 0.0106 & $<0.010$ & 0.020 & 8 & $35 \cdot 8$ & $17 \cdot 0$ & $50 \cdot 0$ & 6 & 0.0550 & $<0.020$ & 0.130 & 7 & 1147 & $<50$ & 4820 \\
\hline Muffin & 8 & 0.0866 & 0.056 & 0.146 & 8 & 11.3 & 7.6 & 19.5 & 8 & 0.0483 & 0.034 & 0.072 & 8 & 4134 & 3530 & 4980 \\
\hline Mushrooms & 4 & 0.0028 & $<0.002$ & 0.008 & 8 & $2 \cdot 1$ & 1.5 & 2.7 & 8 & 0.2161 & 0.132 & 0.336 & 8 & 57 & 46 & 82 \\
\hline Mussels & 8 & 1.5325 & 1.010 & 3.340 & 8 & $88 \cdot 3$ & $32 \cdot 8$ & 154.0 & 8 & 0.5650 & 0.449 & 0.639 & 8 & 4643 & 2790 & 8700 \\
\hline Nectarine & 0 & 0.0010 & $<0.002$ & $<0.002$ & 8 & 1.4 & 0.8 & 1.9 & 0 & 0.0020 & $<0.004$ & $<0.004$ & 0 & 5 & $<10$ & $<10$ \\
\hline Noodles, instant & 7 & 0.0675 & $<0.002$ & 0.186 & 8 & $6 \cdot 0$ & $3 \cdot 0$ & 17.5 & 8 & 0.0291 & 0.005 & 0.055 & 8 & 3074 & 1820 & 4520 \\
\hline Oats, rolled, boiled & 1 & 0.0015 & $<0.002$ & 0.005 & 8 & 8.5 & 6.5 & 9.8 & 6 & 0.0141 & $<0.004$ & 0.033 & 0 & 5 & $<10$ & $<10$ \\
\hline Oil & 0 & 0.0050 & $<0.010$ & $<0.010$ & 4 & $2 \cdot 0$ & $<1.0$ & 4.0 & NR & 0.0010 & 0.001 & 0.001 & 1 & 33 & $<50$ & 90 \\
\hline Onion & 7 & 0.0036 & $<0.002$ & 0.008 & 8 & 1.8 & 1.6 & $2 \cdot 2$ & 3 & 0.0064 & $<0.004$ & 0.021 & 8 & 22 & 12 & 35 \\
\hline Orange, peeled & 1 & 0.0021 & $<0.002$ & 0.010 & 8 & 1.0 & 0.8 & 1.2 & 0 & 0.0020 & $<0.004$ & $<0.004$ & 4 & 14 & $<10$ & 37 \\
\hline Orange juice & 4 & 0.0094 & $<0.001$ & 0.046 & 8 & 0.7 & 0.3 & 1.4 & 0 & 0.0010 & $<0.002$ & $<0.002$ & 7 & 20 & $<5$ & 81 \\
\hline Oysters & 8 & 0.9695 & 0.783 & 1.380 & 8 & 44.0 & $20 \cdot 6$ & 103.0 & 8 & 0.4115 & 0.302 & 0.519 & 8 & 4798 & 2350 & 6910 \\
\hline
\end{tabular}


NS British Journal of Nutrition

Table 3. Continued

\begin{tabular}{|c|c|c|c|c|c|c|c|c|c|c|c|c|c|c|c|c|}
\hline \multirow[b]{2}{*}{ Foods ( $n 8)$} & \multicolumn{4}{|c|}{ lodine (mg/kg) } & \multicolumn{4}{|c|}{$\mathrm{Fe}(\mathrm{mg} / \mathrm{kg})$} & \multicolumn{4}{|c|}{$\mathrm{Se}(\mathrm{mg} / \mathrm{kg})$} & \multicolumn{4}{|c|}{$\mathrm{Na}(\mathrm{mg} / \mathrm{kg})$} \\
\hline & $\begin{array}{l}\text { No. }> \\
\text { LOD }\end{array}$ & Mean & Min & $\operatorname{Max}$ & $\begin{array}{r}\text { No. }> \\
\text { LOD }\end{array}$ & Mean & Min & $\operatorname{Max}$ & $\begin{array}{l}\text { No. }> \\
\text { LOD }\end{array}$ & Mean & Min & $\operatorname{Max}$ & $\begin{array}{l}\text { No. }> \\
\text { LOD }\end{array}$ & Mean & Min & $\operatorname{Max}$ \\
\hline Pasta, boiled & 2 & 0.0149 & $<0.002$ & 0.086 & 8 & $8 \cdot 1$ & 5.0 & $16 \cdot 5$ & 8 & 0.0531 & 0.015 & 0.179 & 2 & 18 & $<10$ & 82 \\
\hline Peaches, canned & 8 & 0.0131 & 0.004 & 0.020 & 8 & 1.9 & 1.1 & $6 \cdot 0$ & 0 & 0.0020 & $<0.004$ & $<0.004$ & 8 & 44 & 19 & 141 \\
\hline Peanut butter & 2 & 0.0513 & $<0.010$ & 0.370 & 8 & $16 \cdot 4$ & $13 \cdot 0$ & $23 \cdot 0$ & 8 & 0.1288 & 0.040 & 0.400 & 8 & 4104 & 1590 & 6100 \\
\hline Peanuts, whole & 3 & 0.0106 & $<0.010$ & 0.030 & 8 & $16 \cdot 3$ & $12 \cdot 0$ & $26 \cdot 0$ & 8 & 0.0625 & 0.030 & 0.140 & 8 & 3269 & 72 & 5830 \\
\hline Pear & 1 & 0.0014 & $<0.002$ & 0.004 & 8 & 1.0 & 0.3 & 1.5 & 0 & 0.0020 & $<0.004$ & $<0.004$ & 2 & 6 & $<10$ & 11 \\
\hline Peas, boiled & 1 & 0.0013 & $<0.002$ & 0.003 & 8 & 16.0 & $12 \cdot 6$ & 27.5 & 0 & 0.0020 & $<0.004$ & $<0.004$ & 3 & 9 & $<10$ & 22 \\
\hline Pineapple, canned & 7 & 0.0150 & $<0.002$ & 0.028 & 8 & $1 \cdot 2$ & 0.6 & $2 \cdot 0$ & 0 & 0.0020 & $<0.004$ & $<0.004$ & 4 & 22 & $<10$ & 68 \\
\hline Pizza, baked & 8 & 0.0309 & 0.017 & 0.060 & 8 & $8 \cdot 1$ & $7 \cdot 0$ & 9.2 & 8 & 0.0653 & 0.047 & 0.092 & 8 & 5914 & 5250 & 7240 \\
\hline Pork chop, fried & 8 & 0.0093 & 0.004 & 0.022 & 8 & 7.9 & $5 \cdot 2$ & $9 . \overline{4}$ & 8 & 0.1373 & 0.098 & 0.183 & 8 & 838 & 731 & 922 \\
\hline Potato crisps & 5 & 0.0131 & $<0.010$ & 0.030 & 8 & 14.6 & 9.0 & 19.0 & 1 & 0.0113 & $<0.020$ & 0.020 & 8 & 3728 & 1990 & 5450 \\
\hline Potato, hot chips & 6 & 0.0700 & $<0.002$ & 0.257 & 8 & 7.3 & 5.6 & 9.2 & 1 & 0.0023 & $<0.004$ & 0.004 & 8 & 2155 & 1300 & 2670 \\
\hline $\begin{array}{l}\text { Potatoes, peeled, } \\
\text { boiled }\end{array}$ & 6 & 0.0028 & $<0.002$ & 0.005 & 8 & 3.1 & $2 \cdot 1$ & 3.8 & 3 & 0.0048 & $<0.004$ & 0.017 & 5 & 17 & $<10$ & 28 \\
\hline $\begin{array}{l}\text { Potatoes, with skin, } \\
\text { microwaved }\end{array}$ & 8 & 0.0109 & 0.002 & 0.027 & 8 & $6 \cdot 0$ & 3.7 & 8.7 & 4 & 0.0066 & $<0.004$ & 0.020 & 5 & 20 & $<10$ & 33 \\
\hline Prunes & 8 & 0.0079 & 0.004 & 0.013 & 8 & $5 \cdot 4$ & 4.3 & $7 \cdot 1$ & 1 & 0.0028 & $<0.004$ & 0.008 & 5 & 19 & $<10$ & 42 \\
\hline Pumpkin, boiled & 3 & 0.0044 & $<0.002$ & 0.013 & 8 & $2 \cdot 3$ & 1.9 & 3.5 & 1 & 0.0024 & $<0.004$ & 0.005 & 0 & 5 & $<10$ & $<10$ \\
\hline Raisins or sultanas & 7 & 0.0167 & $<0.002$ & 0.027 & 8 & 15.1 & 11.8 & 21.5 & 0 & 0.0020 & $<0.004$ & $<0.004$ & 8 & 115 & 40 & 376 \\
\hline Rice, white & 5 & 0.0031 & $<0.002$ & 0.005 & 8 & 0.7 & 0.4 & $1 \cdot 1$ & 5 & 0.0093 & $<0.004$ & 0.021 & 0 & 5 & $<10$ & $<10$ \\
\hline Salad dressing & 5 & 0.0356 & $<0.010$ & 0.170 & 7 & 3.7 & $<1.0$ & $7 \cdot 0$ & NR & 0.0010 & 0.001 & 0.001 & 8 & 6004 & 2190 & 8220 \\
\hline Sausages, fried & 8 & 0.0802 & 0.012 & 0.261 & 8 & 14.5 & $12 \cdot 3$ & $16 \cdot 3$ & 8 & 0.0459 & 0.036 & 0.057 & 8 & 7352 & 6130 & 9180 \\
\hline Silverbeet, boiled & 6 & 0.0270 & $<0.002$ & 0.104 & 8 & 10.4 & 5.4 & 21.9 & 4 & 0.0056 & $<0.004$ & 0.015 & 8 & 716 & 518 & 1130 \\
\hline Snack bars & 6 & 0.1025 & $<0.010$ & 0.490 & 8 & $16 \cdot 3$ & 5.0 & 37.0 & 5 & 0.0275 & $<0.020$ & 0.050 & 8 & 1682 & 640 & 3550 \\
\hline Snacks, flavoured & 8 & 0.0575 & 0.020 & 0.110 & 8 & 9.9 & 3.0 & 14.0 & 5 & 0.0300 & $<0.020$ & 0.060 & 8 & 11569 & 5130 & 21300 \\
\hline Soup, chicken & 8 & 0.0210 & 0.006 & 0.078 & 8 & $1 \cdot 1$ & 0.5 & 2.5 & 4 & 0.0058 & $<0.004$ & 0.019 & 8 & 3660 & 2920 & 4990 \\
\hline Soya milk & 8 & 1.3134 & 0.005 & $9 \cdot 140 \dagger$ & 8 & 3.9 & 1.7 & $6 \cdot 1$ & 8 & 0.0154 & 0.003 & 0.037 & 8 & 561 & 388 & 915 \\
\hline $\begin{array}{l}\text { Spaghetti in sauce, } \\
\text { canned }\end{array}$ & 8 & 0.0199 & 0.013 & 0.039 & 8 & 2.9 & $2 \cdot 2$ & 4.0 & 6 & 0.0095 & $<0.004$ & 0.032 & 8 & 4140 & 3320 & 4790 \\
\hline Strawberries & $2 / 7$ & 0.0021 & $<0.002$ & 0.007 & $7 / 7$ & 2.4 & 1.9 & 3.8 & $0 / 7$ & 0.0020 & $<0.004$ & $<0.004$ & $2 / 7$ & 34 & $<10$ & 109 \\
\hline Sugar & 0 & 0.0050 & $<0.010$ & $<0.010$ & 0 & 0.5 & $<1.0$ & $<1.0$ & 0 & 0.0100 & $<0.020$ & $<0.020$ & 0 & 25 & $<50$ & $<50$ \\
\hline Taro, boiled & 5 & 0.0061 & $<0.002$ & 0.012 & 8 & 6.5 & $2 \cdot 3$ & 8.2 & 0 & 0.0020 & $<0.004$ & $<0.004$ & 5 & 12 & $<10$ & 23 \\
\hline Tea, brewed & 2 & 0.0006 & $<0.001$ & 0.001 & 5 & 0.2 & $<0.1$ & 0.4 & 0 & 0.0010 & $<0.002$ & $<0.002$ & 0 & 3 & $<5$ & $<5$ \\
\hline Tomato & 1 & 0.0011 & $<0.002$ & 0.002 & 8 & 2.1 & 1.4 & $2 \cdot 8$ & 0 & 0.0020 & $<0.004$ & $<0.004$ & 8 & 36 & 16 & 90 \\
\hline Tomato sauce & 8 & 0.0136 & 0.002 & 0.023 & 8 & 4.6 & $2 \cdot 4$ & 6.9 & 2 & 0.0033 & $<0.004$ & 0.007 & 8 & 7073 & 4760 & 8680 \\
\hline Tomatoes in juice & 6 & 0.0055 & $<0.002$ & 0.012 & 8 & $5 \cdot 0$ & $2 \cdot 2$ & $12 \cdot 2$ & 1 & 0.0031 & $<0.004$ & 0.011 & 8 & 1170 & 49 & 2670 \\
\hline Water $\ddagger$ & 7 & 0.0023 & $<0.001$ & 0.005 & 0 & 0.01 & $<0.02$ & $<0.02$ & 0 & 0.0005 & $<0.001$ & $<0.001$ & 8 & 9 & 5.5 & 12.9 \\
\hline $\begin{array}{l}\text { Wheat biscuit cer- } \\
\text { eals }\end{array}$ & 0 & 0.0050 & $<0.010$ & $<0.010$ & 8 & 67.8 & 29.0 & $173 \cdot 0$ & 2 & 0.0313 & $<0.020$ & 0.100 & 7 & 2731 & $<50$ & 3920 \\
\hline Wine, still red & 8 & 0.0090 & 0.002 & 0.014 & 8 & 2.5 & 1.4 & 6.0 & 0 & 0.0010 & $<0.002$ & $<0.002$ & 8 & 39 & 14 & 61 \\
\hline Wine, still white & 7 & 0.0049 & $<0.001$ & 0.012 & 8 & 1.2 & 0.5 & $3 \cdot 1$ & 0 & 0.0010 & $<0.002$ & $<0.002$ & 8 & 41 & 12 & 103 \\
\hline Yeast extract & $6 / 6$ & 0.0552 & 0.020 & 0.090 & 8 & 226.0 & $29 \cdot 9$ & $446 \cdot 0$ & 5 & 0.0172 & $<0.004$ & 0.030 & 8 & 37267 & 32300 & 42000 \\
\hline Yoghurt§ & 8 & 0.0831 & 0.055 & 0.110 & 7 & 0.6 & $<0.2$ & 2.0 & 6 & 0.0059 & $<0.004$ & 0.009 & 8 & 446 & 379 & 512 \\
\hline
\end{tabular}

LOD, limit of detection; IWF, infant weaning food; NR, no result.

Table-ready means as consumed, for example, lettuce on a fresh-weight basis but silverbeet on a boiled-weight basis, banana, peeled (flesh only), fish, filleted and grilled². No salt was added to any foods at the time of cooking. $\dagger$ Anomalous result due to iodine contamination in one brand of soya milk.

† combination of bottled and tap water.
§Fruit-flavoured. 
Table 4. Estimation of mean daily exposure to iodine, iron, selenium and sodium for eight population groups in the 2003-4 New Zealand Total Diet Survey

\begin{tabular}{|c|c|c|c|c|c|c|c|c|}
\hline $\begin{array}{l}\text { Age range (years)... } \\
\text { Nutrient elements }\end{array}$ & $\begin{array}{l}25+ \\
\text { Male }\end{array}$ & $\begin{array}{l}25+ \\
\text { Female }\end{array}$ & $\begin{array}{c}19-24 \\
\text { Young male }\end{array}$ & $\begin{array}{l}11-14^{*} \\
\text { Boy }\end{array}$ & $\begin{array}{c}11-14^{*} \\
\text { Girl }\end{array}$ & $\begin{array}{l}5-6 \dagger \\
\text { Child }\end{array}$ & $\begin{array}{c}1-3 \\
\text { Toddler }\end{array}$ & $\begin{array}{l}\text { 6-12 months } \ddagger \\
\text { Infant }\end{array}$ \\
\hline $\begin{array}{l}\text { lodine exposure (normal-I } \\
\text { soya milk) }(\mu \mathrm{g} / \mathrm{d})\end{array}$ & 85 & 60 & 85 & 60 & 50 & 43 & 47 & 49 \\
\hline $\begin{array}{l}\text { lodine exposure (one high-I } \\
\text { soya milk) }(\mu \mathrm{g} / \mathrm{d}) \S\end{array}$ & 117 & 98 & 99 & 74 & 73 & 57 & 57 & 49 \\
\hline $\operatorname{RDI}(\mu \mathrm{g} / \mathrm{d}) \|$ & 150 & 150 & 150 & 120 & 120 & 90 & 90 & $-\eta$ \\
\hline $\mathrm{Al}(\mu \mathrm{g} / \mathrm{d}) \|$ & - & - & - & - & - & - & - & $110 \emptyset$ \\
\hline$U L(\mu \mathrm{g} / \mathrm{d}) \|$ & 1100 & 1100 & 1100 & 600 & 600 & 300 & 200 & $\star \star$ \\
\hline Fe exposure $(\mathrm{mg} / \mathrm{d})$ & $12 \cdot 8$ & $9 \cdot 1$ & $13 \cdot 3$ & 11.9 & $9 \cdot 3$ & $8 \cdot 0$ & $5 \cdot 8$ & $7 \cdot 1$ \\
\hline $\operatorname{RDI}(\mathrm{mg} / \mathrm{d})$ & 8 & $8-18+\dagger$ & 8 & 8 & 8 & 10 & 9 & 11 \\
\hline UL $(\mathrm{mg} / \mathrm{d})$ & 45 & 45 & 45 & 40 & 40 & 40 & 20 & 20 \\
\hline Se exposure $(\mu \mathrm{g} / \mathrm{d})$ & 67 & 49 & 71 & 55 & 41 & 32 & 21 & 16 \\
\hline RDI $(\mu \mathrm{g} / \mathrm{d})$ & 70 & 60 & 70 & 50 & 50 & 30 & 25 & -9 \\
\hline $\mathrm{Al}(\mu \mathrm{g} / \mathrm{d})$ & - & - & - & - & - & - & - & 159 \\
\hline$U L(\mu \mathrm{g} / \mathrm{d})$ & 400 & 400 & 400 & 280 & 280 & 150 & 90 & 60 \\
\hline $\mathrm{Na}$ exposure $(\mathrm{mg} / \mathrm{d})$ & 3047 & 2150 & 3603 & 3108 & 2496 & 2031 & 1384 & 845 \\
\hline $\mathrm{UL}(\mathrm{mg} / \mathrm{d})$ & 2300 & 2300 & 2300 & 2000 & 2000 & 1400 & 1000 & ** \\
\hline
\end{tabular}

RDI, recommended daily intake; UL, upper limit; AI, adequate intake.

* Nutrient reference values for 11-14-year-old children assumed equivalent to values for 9-13-year-old children.

† Nutrient reference values for 5-6-year-old children assumed equivalent to values for 4-8-year-old children.

$\ddagger$ Nutrient reference values for 6-12-month-old infants extrapolated from values for 7-12-month-old infants.

$\S$ Includes anomalous result due to iodine contamination in one brand of soya milk.

$\|$ Australian and New Zealand Nutrient Reference Values ${ }^{8}$.

१ Al, not RDI.

** Unable to be set.

††RDI for women under 50 years is $18 \mathrm{mg} / \mathrm{d}$; RDI for women over 50 years is $8 \mathrm{mg} / \mathrm{d}$.

exposure varied from a low of only $40 \%$ of the RDI, for a $25+$-year-old female, to a high of $57 \%$ of the RDI for a $25+$-year-old male. When the anomalously high levels of iodine found in one product line in one of four brands of soya milk were included, the exposures increased accordingly but all remained well below the RDI.

It is important to note that consistent with previous NZTDS, the 2003-4 NZTDS did not take into account the addition of discretionary salt used either during cooking, or added at the table. Given that over $80 \%$ of discretionary salt is iodised in New Zealand ${ }^{31}$, actual iodine exposures of New Zealanders are probably higher than those indicated in Table 4.

Dairy foods were a dominant source of iodine exposure for all population groups, some of which are shown in Fig. 1. Other animal sources (eggs, mussels, fresh fish and oysters) were also significant to iodine exposure for a 19-24-yearold young male, a $25+$-year-old male and female and an 11-14-year-old boy and girl. Males (aged 19+ years) gained $5-6 \%$ of iodine exposure from beer consumption.
Exposure of iodine for a 6-12-month-old infant was dominated by levels in infant and follow-on formula.

Iron. Estimated dietary exposures to $\mathrm{Fe}$ were above the RDI for $25+$-year-old males, 19-24-year-old young men, and 11-14-year-old boys and girls living in New Zealand. However, the average exposure to $\mathrm{Fe}$ for $25+$-year-old females was only about half the RDI. Fe exposures for a 5-6-year-old child, a 1-3-year-old toddler and 6-12month-old infants were also below the RDI. Mean $\mathrm{Fe}$ exposures for all age-sex groups in the NZTDS were within the range of $20-36 \%$ of upper intake limits and therefore do not represent a toxicological risk from too much $\mathrm{Fe}$.

Food groups and specific foods that contributed to the $\mathrm{Fe}$ exposure for the 25+-year-old male, 1-3-year-old toddler and 6-12-month-old infant are shown in Fig. 2. Grain products and red meat (beef and lamb) were important contributors to Fe exposure for $25+$-year-old males and females, 19-24-year-old young males and 11-14-year-old boys and girls. Breakfast cereals (particularly wheat biscuit cereals
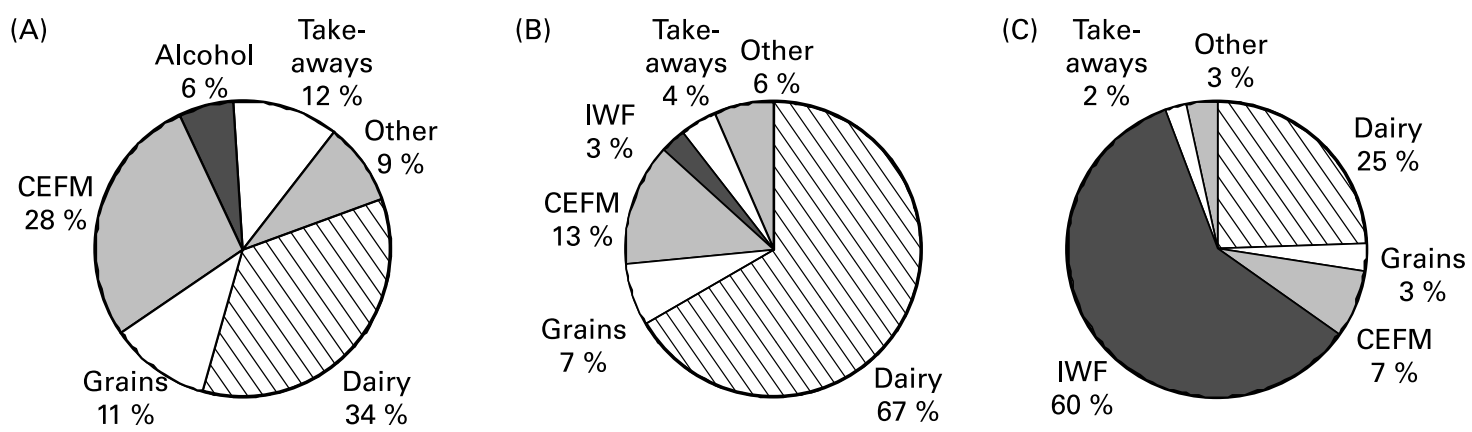

Fig. 1. Food groups that contribute to estimated iodine exposure for (A) a 19-24-year-old young male, (B) a 1-3-year-old toddler and (C) a 6-12-month-old infant in the 2003-4 New Zealand Total Diet Survey. CEFM, chicken, eggs, fish and meat; IWF, infant formulae and weaning foods. 

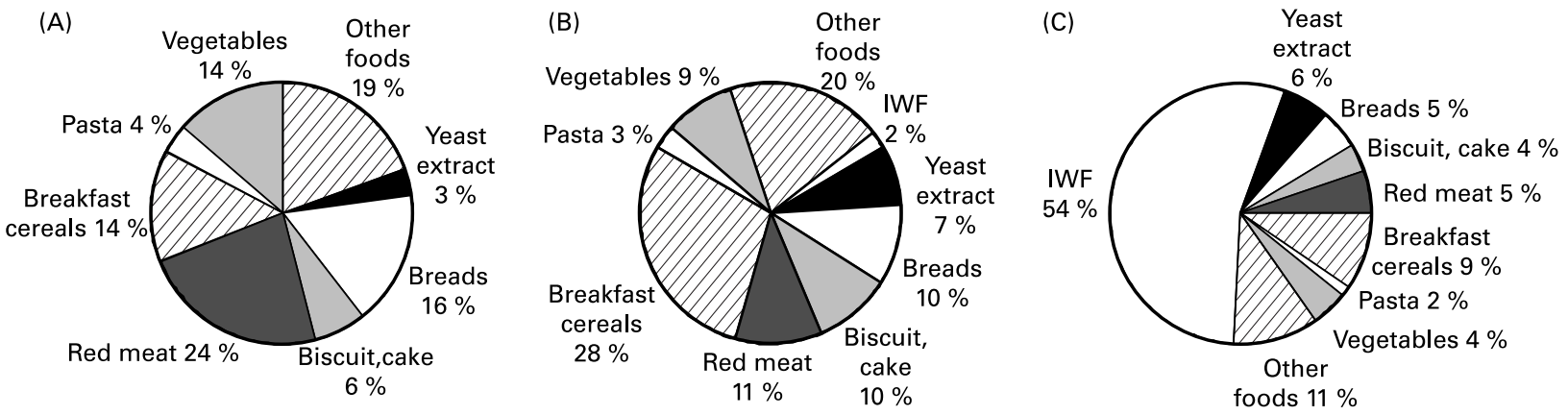

Fig. 2. Food groups that contribute to estimated dietary Fe exposure in (A) a $25+$-year-old male, (B) a 1-3-year-old toddler and (C) a 6-12-month-old infant in the 2003-4 New Zealand Total Diet Survey. IWF, infant formulae and weaning foods.

and cornflakes), yeast extract and breads were the major contributors to the exposure of a 1-3-year-old toddler; and infant formula and weaning foods, yeast extract and breakfast cereals (largely wheat biscuit cereals) are the four major contributing foods for a 6-12-month-old infant.

Selenium. The estimated exposure for a $25+$-year-old female was $49 \mu \mathrm{g} / \mathrm{d}, 18 \%$ below the RDI of $60 \mu \mathrm{g} / \mathrm{d}$. The mean exposure for an 11-14-year-old boy was above the RDI, whereas for an 11-14-year-old girl it was $18 \%$ below, attributable to a lower consumption of fresh fish. Mean exposures of Se for a 19-24-year-old young male, $25+$-year-old male, 5-6-year-old child, 1-3-year-old toddler and 6-12-month-old infant approximated the RDI.

An exposure of up to $400 \mu \mathrm{g}$ Se per d is considered safe for adults, with correspondingly lower upper limits for younger individuals ${ }^{8}$. The 2003-4 NZTDS confirms that Se exposures, on average, do not constitute a toxicity problem for any of the New Zealand age-sex groups.

Seafood, chicken, eggs, breads and grain products provided the majority of Se in the diets of all age-sex groups included in the 2003-4 NZTDS except for the 6-12-month-old infant for whom infant weaning foods contributed nearly $30 \%$. Fig. 3 illustrates the sources of Se in the diet of a 19-24-year-old young male (also representative of a $25+$-year-old female and a $25+$-year-old male), a 1-3-year-old toddler and a 6-12-month-old infant. Adolescent boys and girls (age 11-14 years) showed lower contributions from fish (both fresh and battered) and cake than their adult counterparts but higher contributions from chicken and white bread, reflecting a preference for these foods by these population groups. Chicken feed and supplements specifically include Se and that accounts for eggs as a key contributor to Se exposure (E. Catherwood, Executive
Officer, Poultry Industry Association of New Zealand, Egg Producers Federation Of New Zealand and New Zealand Feed Manufacturers Association, personal communication, 1999, N. Meads, Nutritionist, Tegel Foods Poultry manufacturer, personal communication, 1999).

Sodium. Mean daily $\mathrm{Na}$ exposures were significantly above the acceptable exposure for all age-sex groups, and exceeded the upper intake limits for $25+$-year-old males, 19-24-year-old young males and 11-14-year-old boys and girls by up to $125 \%$ for the average consumer. For a high consumer (95th percentile), exposure to $\mathrm{Na}$ could be approximately twice that of an average consumer ${ }^{30}$, resulting in $\mathrm{Na}$ exposures up to $6200 \mathrm{mg} / \mathrm{d}$ for the $11-14$-year-old boy, more than three times the upper intake limit.

The daily exposure estimates in Table 4 included only $\mathrm{Na}$ inherent in the food, and $\mathrm{Na}$ added at the time of food manufacture, or preparation of takeaways, but not salt added at the time of cooking or at the table. $\mathrm{Na}$ inherent in foods has been estimated to contribute about $15 \%$, and Na from manufactured or processed foods $65-70 \%$ of total $\mathrm{Na}$ exposure ${ }^{13,32}$. Salt added at the time of cooking or at the table has been estimated to account for $11-20 \%$ of total $\mathrm{Na}$ exposure ${ }^{13,32,33}$. Hence, if this salt is included, $\mathrm{Na}$ exposure may be up to $20 \%$ higher than the values stated in Table 4.

Exposures of $\mathrm{Na}$ for an average New Zealand consumer range from $845 \mathrm{mg} / \mathrm{d}$ for a 6-12-month-old infant to $3603 \mathrm{mg} / \mathrm{d}$ for a $19-24-$ year-old young male. Blood pressure increases in a dose-dependent way with $\mathrm{Na}$ intakes from $230 \mathrm{mg} / \mathrm{d}^{8}$. Therefore it is likely that $\mathrm{Na}$ in the average New Zealand diet is causing elevated blood pressure. Elevated blood pressure is a risk factor for $\mathrm{CVD}^{8}$ and $\mathrm{CVD}$ is a leading cause of death in New Zealand ${ }^{34}$.
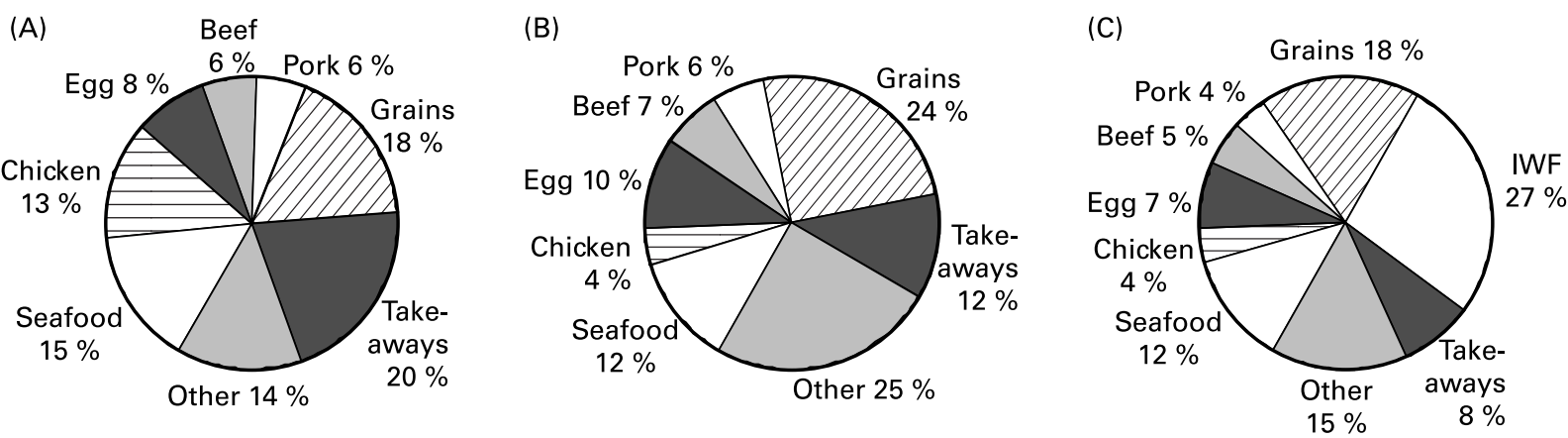

Fig. 3. Foods that contribute to estimated dietary Se exposure in (A) a 19-24-year-old young male, (B) a 1-3-year-old toddler and (C) a 6-12-month-old infant in the 2003-4 New Zealand Total Diet Survey. IWF, infant formulae and weaning foods. 


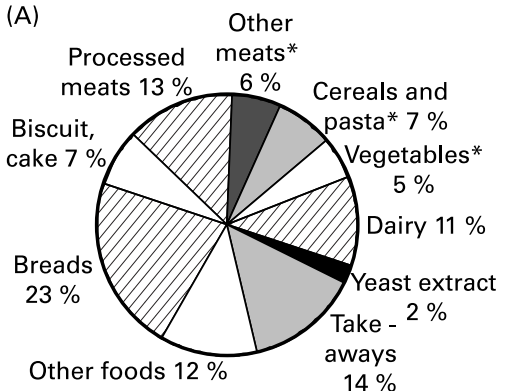

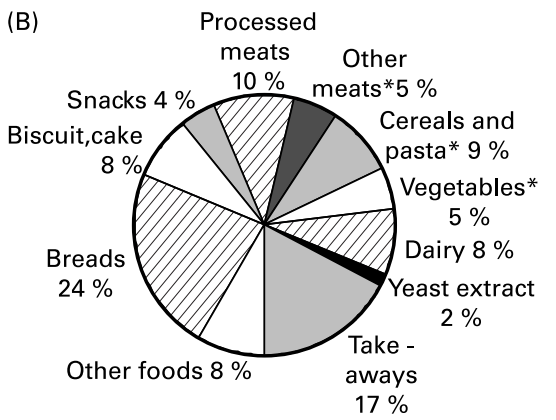

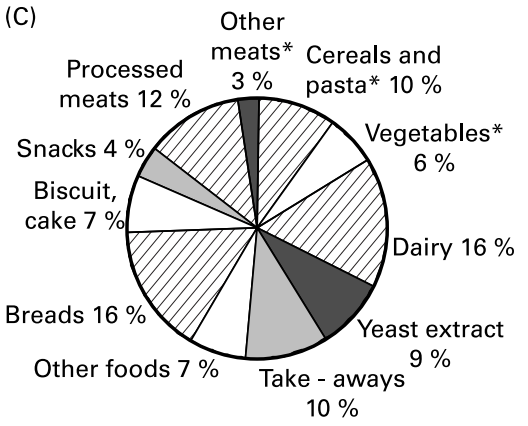

Fig. 4. Foods that contribute to estimated dietary $\mathrm{Na}$ exposure in (A) a $25+$-year-old male, (B) an 11-14-year-old girl and (C) a 6-12-month-old infant in the 2003-4 New Zealand Total Diet Survey, excluding salt added at time of cooking or at the table. * Contribution of these foods to Na intake will increase if salt is added at the time of cooking or at the table.

The distribution of foods contributing to Na exposure for an adult male, an adolescent girl, and an infant, as representatives of the eight population groups, is shown in Fig. 4. Of $\mathrm{Na}$ exposure, 80 to $86 \%$ was attributable to forty-two manufactured foods with the single greatest contributor to $\mathrm{Na}$ exposure being bread, accounting for $15-27 \%$, followed by processed meats (bacon, ham, corned beef and sausages), contributing $10-14 \%$ of total $\mathrm{Na}$ exposure. Grain products collectively account for $33-48 \%$ of $\mathrm{Na}$ exposure, similar to the estimate of $38 \%$ from the UK National Food Survey ${ }^{32}$.

\section{Exposure trends}

The impacts of increasing globalisation of the food supply and changes in consumption patterns on nutrient exposure are apparent from successive NZTDS undertaken in New Zealand over the 20-year period from 1982 (Fig. 5).

The iodine exposure of New Zealanders has continued to decrease for all age-sex groups, to a point where iodine

(A)

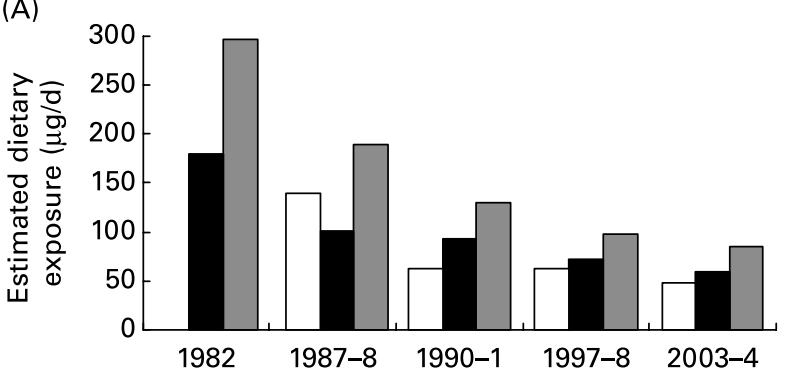

(C)

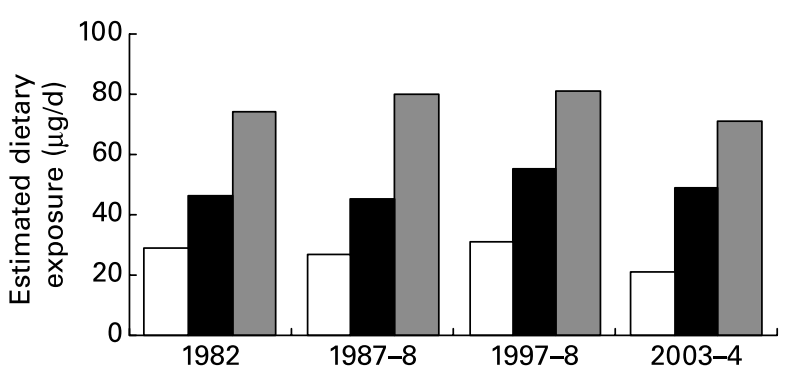

exposures are now, at best, only $57 \%$ of the RDI across the eight population groups. The reduction in dietary iodine exposures is consistent with New Zealand studies showing urinary iodide levels, and hence iodine status, to be low and decreasing ${ }^{24,35-38}$. This is a significant public health issue for New Zealand and the appropriate action to address the low dietary exposure is under consideration by the New Zealand government. The observed downward trend in iodine exposure is at least partly explained by the marked drop in the use of iodophor disinfectants in the dairy industry in the period 1978-88 and changing consumption patterns. Sutcliffe ${ }^{39}$ reported a dramatic decrease in iodine concentration of milk from $87 \mu \mathrm{g} / 200 \mathrm{ml}(0.44 \mathrm{mg} / \mathrm{kg})$ in 1978 to $24 \mu \mathrm{g} / 200 \mathrm{ml}(0 \cdot 12 \mathrm{mg} / \mathrm{kg})$ in 1988 that accounts for the decrease in exposure seen between 1982 and 1987-8 (Fig. 5). The contribution of the top ten individual foods to iodine exposure in 2003-4 is shown in Table 5 along with the concentration and consumption of these same foods from previous NZTDS, where these are available. Data for individual foods

(B)
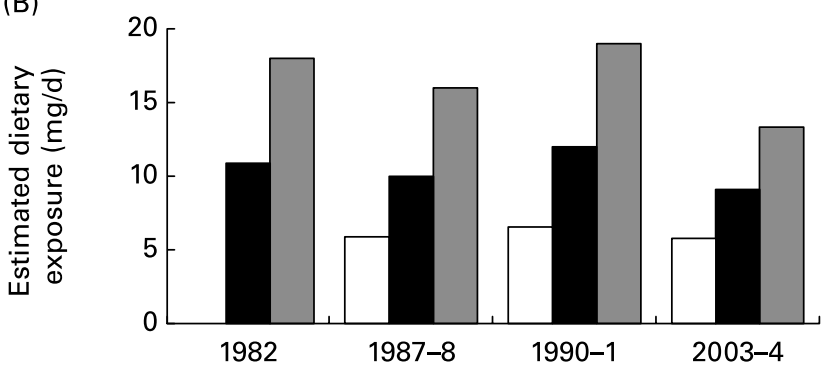

(D)

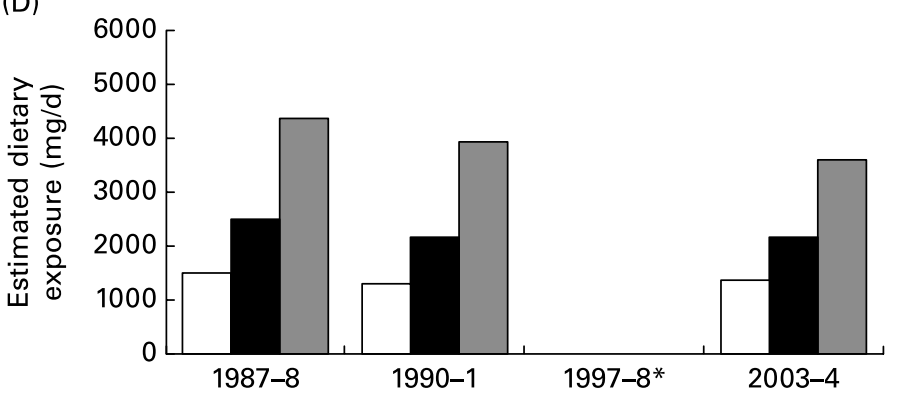

Fig. 5. Trends of estimated dietary exposure to iodine (A), Fe (B), Se (C) and Na (D) for a 19-24-year-old young male ( $\square$ ), a $25+-$ year-old female ( $\square$ ) and a $1-$ 3-year-old toddler $(\square)$ over five New Zealand Total Diet Surveys from 1982 to $2003-4^{3,9-11}$. * Na was not included in the analytes of the $1997-8$ New Zealand Total Diet Survey. 
Table 5. Concentration of iodine and consumption of iodine-contributing foods for successive total diet surveys for a young male

\begin{tabular}{|c|c|c|c|c|c|c|c|c|}
\hline \multirow{2}{*}{$\begin{array}{l}\text { Food } \\
\text { Year range... }\end{array}$} & \multirow{2}{*}{$\frac{\text { Exposure (\%) }}{2003-4}$} & \multicolumn{4}{|c|}{ lodine concentration (mg/kg) } & \multicolumn{3}{|c|}{ Consumption $(g / d)$} \\
\hline & & $2003-4$ & $1997-8$ & $1990-1$ & $1987-8$ & $2003-4$ & $1997-8$ & $1990-1$ \\
\hline Milk, 3.25 and $0.5 \%$ fat & $28 \cdot 8$ & 0.09 & 0.09 & 0.06 & $0 \cdot 10$ & 266 & 365 & 426 \\
\hline Eggs & $12 \cdot 7$ & 0.52 & 0.54 & 0.16 & 0.25 & 21 & 17 & 31 \\
\hline Cake & $5 \cdot 9$ & 0.13 & 0.08 & 0.17 & 0.19 & 39 & 34 & 35 \\
\hline Beer & $5 \cdot 4$ & 0.01 & NR & 0.01 & 0.03 & 364 & 188 & 268 \\
\hline Potato, hot chips & 4.9 & 0.07 & 0.35 & 0.03 & 0.01 & 59 & 26 & 36 \\
\hline Mussels & 3.9 & 1.53 & 1.52 & NR & NR & 2 & 2.5 & NR \\
\hline Fish, fresh & 3.6 & 0.22 & 0.38 & 0.28 & 0.21 & 14 & 5 & 14 \\
\hline Fish, battered & $2 \cdot 8$ & 0.17 & 0.18 & 0.08 & 0.13 & 14 & 10 & 30 \\
\hline Oysters & 2.4 & 0.97 & 1.05 & 0.26 & 0.67 & 2 & 2.5 & 12 \\
\hline Chocolate, plain milk & $2 \cdot 2$ & 0.15 & 0.11 & 0.13 & 0.09 & 12 & 3 & $3 \cdot 6$ \\
\hline
\end{tabular}

NR, no result.

were not obtained before the 1987-8 NZTDS. The decreasing exposure to iodine (since 1987-8) is reflected in the decrease in consumption, for a young male, of milk and eggs rather than a change in iodine concentrations in these foods.

A comparison of mean $\mathrm{Fe}$ exposure data for a $25+$-yearold female and 19-24-year-old young male over the 20-year period from 1982 suggests that exposure for $25+$-year-old females has changed little over this period. There is, however, a marked decline in exposure for a 19-24-year-old young male between 1990-1 and 2003-4. The reason for this is not apparent since a reduced exposure from wholemeal bread and lamb's liver is more than offset by increases from fortified cereals and takeaways.

Dietary exposures of Se have been steady over the 20-year period from 1982 to $2003-4$. This finding is at variance with a reported increase in Se status of New Zealanders ${ }^{40,41}$ that was attributed to an increased consumption of wheat imported from areas richer in Se, such as Australia and/or North America, and the use of livestock supplements ${ }^{3}$. The apparent increase in $\mathrm{Se}$ exposure estimate for the $25+$-year-old female and 1-3-year-old toddler reported in the 1987-8 NZTDS has not been maintained.

Na was not included in the analytes of the 1997-8 NZTDS but exposures for a young male, a $25+$-year-old female and a 1-3year-old toddler have decreased by 17,14 and $8 \%$ respectively since 1987. However, the 2003-4 NZTDS showed that mean daily $\mathrm{Na}$ exposures exceed the adequate intake range for all age-sex groups and exceed the upper intake limit for a $25+$-year-old male, a 19-24-year-old young male, an 11-14-year-old boy and girl, a 5-6-year-old child and a 13 -year-old toddler by $25-57 \%$. For a high consumer, $\mathrm{Na}$ exposure may exceed the upper intake limit by a factor of 3 . None of these estimates include the use of discretionary salt that may also increase $\mathrm{Na}$ exposure by up to an additional $20 \%$.

\section{Conclusion}

The NZTDS methodology is internationally accepted with similar studies conducted in the $\mathrm{USA}^{42}$, Australia ${ }^{27}, \mathrm{China}^{43,44}$, $\mathrm{UK}^{45}$, Czechoslovakia ${ }^{46}$ and France ${ }^{29}$. These studies allow an assessment of the chemical safety of the food supply and identification of exposure concerns for regulatory action, further research, or education, for improved public health. The 20034 NZTDS reveals that the calculated dietary iodine exposures for all age-sex groups are well below recommended levels and have steadily decreased since 1982 . This is of concern to public health, especially the physical and mental development of infants and young children. Fe exposures are adequate for the average male and 11-14 year olds. However, despite the fortification of cereals, the average exposure to $\mathrm{Fe}$ in New Zealand for a $25+$-year-old female is only about half the RDI. Calculated mean daily dietary exposures of Se for all age-sex groups of the 2003-4 NZTDS meet or are slightly below the RDI, with those living in the South Island and in low-Se areas likely to have lower dietary exposures of Se. Despite more global distribution of food, mean Se exposures have been steady in the past 20 years. Mean daily $\mathrm{Na}$ exposures exceed the adequate intake range for all age-sex groups and exceed the upper intake limit for most groups. Given that the majority of $\mathrm{Na}$ exposure is from processed foods, these are possible foods to target for $\mathrm{Na}$ reduction.

\section{Acknowledgements}

The present study was undertaken by the Institute of Environmental Science and Research Ltd as part of their contractual agreement with the New Zealand Food Safety Authority. The authors gratefully acknowledge the contributions of Cherie Flynn, project leader from the NZFSA, Health Protection Officers at Auckland District Health Board, Hawkes Bay District Health Board, Crown Public Health and Public Health South for food sampling, Mrs Shirley Jones, Miss Hui-Ming Lin and Miss Kate Thomas for preparing foods and Mr Peter Cressey, ESR, for his contribution to the process for calculating estimated dietary exposures.

\section{References}

1. Thomson CD (2003) The declining iodine status of New Zealanders: reasons, consequences and possible solutions. Proc Nutr Soc N Z 28, 35-42.

2. Thomson CD (2004) Selenium and iodine intakes and status in New Zealand and Australia. B J Nutr 91, 661-672.

3. Vannoort RW, Cressey PJ \& Silvers K (2000) 1997/98 New Zealand Total Diet Survey Part 2, Elements. Wellington: Ministry of Health, http://www.moh.govt.nz/publications.

4. Hetzel BS \& Maberly GF (1986) Iodine. In Trace Elements in Human and Animal Nutrition, 5th ed. pp., 139-208 [W Mertz, editor]. Orlando, FL: Academic Press, vol. 2. 
5. Mann J \& Truswell S (1998) Essentials of Human Nutrition Oxford: Oxford University Press.

6. Hoekstra WG (1975) Biochemical function of selenium and its relation to vitamin E. Fed Proc 34, 2083-2089.

7. Garland M, Stampfer MJ, Willett WC \& Hunter DJ (1994) The epidemiology of selenium and human cancer. In Natural Antioxidants in Human Health and Disease, pp. 263-286 [B Frei, editor]. San Diego, CA: Academic Press.

8. National Health and Medical Research Centre and Ministry of Health (2006) Nutrient Reference Values for Australia and New Zealand Including Recommended Dietary Intakes. Canberra, Australia: National Health and Medical Research Council, Wellington, NZ: Ministry of Health.

9. Pickston L, Brewerton HV, Drysdale JM, et al. (1985) The New Zealand diet: a survey of elements, pesticides, colours, and preservatives. $N Z J$ Tech 1, 81-89.

10. Institute of Environmental Science and Research Ltd and Ministry of Health (1994) The 1987/88 New Zealand Total Diet Survey. Wellington, NZ: Ministry of Health.

11. Hannah ML, Vannoort RW \& Pickston L (1995) 1990/91 New Zealand Total Diet Survey, Part 3: Nutrients. ESR Client Report FW95. Wellington, NZ: ESR.

12. Fregly MJ (1984) Sodium and potassium. In Nutrition Reviews' Present Knowledge in Nutrition, 5th ed. pp., 439-458 Washington, DC: The Nutrition Foundation.

13. Mattes RD \& Donnelly D (1991) Relative contributions of dietary sodium sources. J Am College Nutr 19, 383-393.

14. 10th ed. National Research Council and Food and Nutrition Board (1989) National Research Council and Food and Nutrition Board. Washington, DC: National Academy Press.

15. World Health Organization (2006) Food Safety. International Workshops for Total Diet, Studies at Kansas City, 1999, Brisbane, 2002 and Paris, 2004. http://www.who.int/foodsafety/ publications/chem/en/index.html.

16. Dick GL, Hughes JT, Mitchell JW \& Davidson F (1978) Survey of trace elements and pesticide residues in the New Zealand diet. 1 Trace element content. $N$ Z J Sci 21, 57-69.

17. Dick GL, Heenan JL, Love JL, Udy PB \& Davidson F (1978) Survey of trace elements and pesticide residues in the New Zealand diet. 2 Organochlorine and organophosphorus pesticide residue content. N Z J Sci 21, 71-78.

18. Vannoort RW, Hannah ML, Pickston L \& Fry JM (1995) 1990/91 New Zealand Total Diet Survey, Part 1: Pesticide Residues. ESR Client Report FW 95/6. Wellington, NZ: Ministry of Health.

19. Vannoort RW, Hannah ML, Pickston L \& Fry JM (1995) 1990/ 91 New Zealand Total Diet Survey, Part 2: Contaminant Elements. ESR Client Report FW 95/7. Wellington, NZ: Ministry of Health.

20. Cressey PJ, Vannoort RW, Silvers K \& Thomson B (2000) New Zealand Total Diet Survey, Part 1: Pesticide Residues. Wellington, NZ: Ministry of Health, http://www.moh.govt.nz/publications.

21. Vannoort RW \& Thomson BM (2005) 2003/04 New Zealand Total Diet Survey. Wellington, NZ: New Zealand Food Safety Authority, www.nzfsa.govt.nz/science/research-projects/totaldiet-survey/reports/full-final-report/index.htm.

22. Brinsdon S (2004) Simulated typical diets for the 2003/2004 New Zealand Total Diet Survey. A Report for the New Zealand Food Safety Authority, http://nzfsa.govt.nz/science-technology/ research-projects/total-diet-survey/index.htm.

23. Russell DG, Parnell WR, Wilson NC, et al., NZ Food: NZ People. Key Results of the 1997 National Nutrition Survey, Wellington, NZ: Ministry of Health.

24. Ministry of Health (2003) NZ Food, NZ Children: Key Results of the 2002 National Children's Nutrition Survey. Wellington, NZ: Ministry of Health.

25. Soh P, Ferguson EL, McKenzie JE, Skeaff S, Parnell W \& Gibson RS (2002) Dietary intakes of 6-24-month-old urban
South Island New Zealand children in relation to biochemical iron status. Public Health Nutr 5, 339-346.

26. Fecher PA, Goldmann I \& Nagengast A (1998) Determination of iodine in food samples by inductively coupled plasma mass spectrometry after alkaline extraction. J Anal At Spectrom 13, 977-982.

27. Food Standards Australia New Zealand (2003) Food Standards Australia New Zealand. In The 20th Australian Total Diet Study. Canberra: Food Standards Australia New Zealand.

28. Capar SG \& Cunningham WC (2000) Element and radionuclide concentrations in food: FDA Total Diet Study, 19911996. JAOAC 83, 157-177.

29. Leblanc J-C, Guérin T, Noël L, Calamassi-Tran G, Volatier JL \& Verger P (2005) Dietary exposure estimates of 18 elements from the 1st French Total Diet Study. Food Add Contam 22, 624-641.

30. Food and Agriculture Organization, United Nations Environment Programme and World Health Organization (1985) Guidelines for the Study of Dietary Intake of Chemical Contaminants - Report of the Joint FAO/UNEP/WHO Food Contamination Monitoring Programme, WHO Offset Publication no. 87. Geneva: WHO.

31. Shannon C (2004) Introduction of Salt to Infant and Toddler Diets - Attitudes and Practices of Parents and Caregivers. Dietetics Practicum. Dunedin, NZ: University of Otago.

32. Scientific Advisory Committee on Nutrition (2003) Salt and Health. Norwich: The Stationery Office, http://www.food.gov. uk/multimedia/pdfs/saltandhealth0503.pdf.

33. Institute of Food Science and Technology (2003) Salt http:// www.ifst.org/hottop17.htm

34. New Zealand Health Information Service (2006) Cancer Registrations and Deaths 2002. Wellington, NZ: Ministry of Health.

35. Skeaff SA, Ferguson EL, McKenzie JE, Valeix P, Gibson RS \& Thomson CD (2005) Are breast-fed infants and toddlers in New Zealand at risk of iodine deficiency? Nutrition 21, 325-331.

36. Thomson CD, Packer MA, Butler JA, Duffield AJ, O'Donaghue KL \& Whanger PD (2001) Urinary selenium and iodine during pregnancy and lactation. J Trace Elem Med Biol 14, 210-217.

37. Thomson CD, Colls AJ, Conaglen JV, Macormack M, Stiles M \& Mann J (1997) Iodine status of New Zealand residents as assessed by urinary iodide excretion and thyroid hormones. Br J Nutr 78, 901-912.

38. Thomson CD, Packer MA, Butler JA, Duffield AJ, O'Donaghue KL \& Whanger PD (2001) Urinary selenium and iodine during pregnancy and lactation. J Trace Elem Med Biol 14, 210-217.

39. Sutcliffe E (1990) Iodine in New Zealand milk. Food Tech N Z 25, 32-34.

40. Winterbourn CC, Saville DJ, George PM \& Walmsley TA (1992) Increase in selenium status of Christchurch adults associated with deregulation of the wheat market. $N Z$ Med $J \mathbf{1 0 5}$, $466-468$.

41. Thomson CD \& Robinson MF (1996) The changing selenium status of New Zealand residents. Eur J Clin Nutr 50, 107-114.

42. Egan SK, Tao SS-H, Pennington JAT \& Bolger PM (2002) US Food and Drug Administration's Total Diet Study: intake of nutritional and toxic elements, 1991-96. Food Addit Contam 19, $103-125$.

43. Chen J \& Gao J (1993) The Chinese Total Diet Study in 1990, part I. Chemical contaminants. JAOAC Int 76, 1193-1205.

44. Chen J \& Gao J (1993) The Chinese Total Diet Study in 1990, part II. Nutrients. JAOAC Int 76, 1206-1213.

45. Food Safety Authority (2004) Survey of metals and other elements. Food Survey Information sheet 48/04 http://www. food.gov.uk/science/surveillance

46. Ruprich J (2003) The 2002 Total Diet Study of the Czech Republic http://www.chpr.szu.cz/monitor/tds02c/tds02c.htm

47. Brinsdon S (1999) Simulated typical diets for the 1997/98 New Zealand Total Diet Survey. Wellington: Ministry of Health. 